\title{
Correlação entre os niveis plasmáticos de apolipoproteínas A-I e B e o perfil lipídico em indivíduos com e sem diabetes mellitus tipo 2 e hipertensão arterial
}

\author{
Apolipoproteins A-I and B plasma levels correlations with lipid profile in subjects with type 2 diabetes mellitus \\ and high blood pressure
}

Luciana Moreira Lima'; Maria das Graças Carvalho²; Anna Letícia Soares'; Marcelo Carvalho Lasmar; Bethânia Alves Novelli; ; Marinez Oliveira Sousa ${ }^{2}$

\begin{abstract}
unitermos Perfil lipidico

Apolipoproteinas

Diabetes mellitus tipo 2

Hipertensão arterial

\section{resumo}

Introdução: As dislipidemias, o diabetes mellitus (DM) e a hipertensão arterial sistêmica (HAS) são importantes fatores no desenvolvimento da doença arterial coronariana (DAC), a principal causa de morte de indivíduos adultos no mundo. Diversos estudos têm demonstrado correlação positiva entre concentrações plasmáticas elevadas de colesterol presente na lipoproteína de baixa densidade (LDL-C) e/ou baixas concentrações de colesterol presente na lipoproteína de alta densidade (HDL-C) e aumento de risco para doenças cardiovasculares (DCV). Objetivo: Correlacionar os valores do perfil lipídico, apolipoproteínas (Apo) A-I e B, lipoproteína(a) [Lp(a)] e microalbuminúria em indivíduos com e sem DM e HAS. Material e métodos: Os participantes, com faixa etária de 40 a 65 anos, foram divididos em cinco grupos: 1 . controle (indivíduos hígidos, $n=16) ; 2$. HAS (hipertensos, $n=12) ; 3$. DM (DM2, normotensos e normoalbuminúricos, $n=7) ; 4$. $\mathrm{DM}+\mathrm{HASnAlb}$ (DM2, hipertensos e normoalbuminúricos, $n=18)$; e 5. DM + HASmAlb (DM2, hipertensos e microalbuminúricos, $n=9)$. Resultados: Foram observadas diferenças estatísticas para o LDL-C entre a média do grupo 5 em relação às médias dos demais grupos; para triglicérides (TCL), entre as médias dos grupos 2,4 e 5 em relação à do grupo 1. Para as médias de colesterol total (CT), HDL-C, Lp(a) e Apo A-I não houve diferença significativa entre os grupos. As médias para Apo B dos grupos 4 e 5 apresentaram diferenças significativas com relação ao grupo 1. Foi observada correlação positiva entre o $L D L-C$ e a $A p o B(r=0,684)$; $p<0,0001)$ e entre o HDL-C e a Apo A-I $(r=0,374 ; p=0,003)$, de acordo com a literatura. Conclusão: Entre todos os parâmetros avaliados o LDL-C foi o único que apresentou diferenças significativas entre o grupo 5 , cujos indivíduos apresentam as duas patologias combinadas (DM e HAS), além da microalbuminúria, em relação aos demais grupos estudados, sugerindo que a associação das duas patologias, aliada à presença de microalbuminúria, poderia ter contribuído para o agravamento da dislipidemia.
\end{abstract}

Background: Dyslipidemias, diabetes mellitus (DM), high blood pressure are important factors for development of the coronary artery disease (CAD), principal cause of death in the world. Several studies have demonstrated positive correlation between both LDL-C high plasma levels and HDL-C low concentrations and increased risk for cardiovascular diseases. Objectives: To establish the possible correlation between lipids, lipoproteins, apolipoproteins A-I and B, Lipoprotein (a) and microalbuminuria in subjects with and without diabetes mellitus type 2 and high blood pressure. Material and Method: The subjects, with age range from 40 to 65 years, were divided into five groups: 1. control (normal subjects, $\mathrm{n}=16$ ); 2. HAS (subjects with high blood pressure, $\mathrm{n}=12$ ); 3. DM (normotensive and normoalbuminuric patients with diabetes mellitus type $2, \mathrm{n}=7$ ); $4 . D M+H A S n A l b$ (hypertensive and normoalbuminuric patients with diabetes mellitus type $2, \mathrm{n}=18$ ) and $5 . D M+H A S m A l b$ (hypertensive and microalbuminuric patients with diabetes mellitus type 2, $n=9$ ). Results: Concerning to the lipid profile statistical differences were observed for LDL-C between the average of the group 5 compared to the averages of the other groups; for triglycerides among the groups 2, 4 and 5 compared to the group 1. For the total cholesterol, HDL-C, Lp(a) and Apo A-I no significant difference was observed among the groups. Average values for Apo $B$ for groups 4 and 5 presented significant difference compared to group 1. Positive correlation was observed between $L D L-C$ and $A p o B(r=0,684) ; p<0,0001)$ and HDL-C and Apo A-I $(r=$ $0,374 ; p=0,003)$, according to literature. Conclusion: Among all parameters assessment, $L D L-C$ was the unique one that showed significant differences between the group 5, whose participants present both alterations (DM and HAS) besides the microalbuminuria, related to other groups studied. This comes to suggest that the association between the two diseases allied to microalbuminuria may contribute to the dyslipidemia aggravation. key words

Lipid profile

Apolipoproteins

Type 2 diabetes mellitus

High Blood Pressure

1. Estudante de pós-graduação, nível doutorado, da Faculdade de Farmácia da Universidade Federal de Minas Cerais (UFMG).

2. Doutora do Departamento de Análises Clínicas e Toxicológicas da Faculdade de Farmácia da UFMG.

3. Acadêmico de Farmácia; bolsista de Iniciação Científica da Faculdade de Farmácia da UFMG.

4. Médica angiologista da Santa Casa de Misericórdia de Belo Horizonte (SCBH).

Trabalho realizado no Departamento de Análises Clínicas e Toxicológicas da Faculdade de Farmácia da Universidade Federal de Minas Cerais (UFMG) e apresentado sob a forma de pôster no 38

Congresso Brasileiro de Patologia Clínica/Medicina Laboratorial, Florianópolis/2004. 


\section{Introdução}

O diabetes mellitus (DM) é um distúrbio que acomete aproximadamente 150 milhões de pessoas no mundo, comprometendo-lhes a qualidade de vida. Aproximadamente $90 \%$ dos casos de DM são do tipo $2^{(33)}$. A hipertensão arterial sistêmica (HAS) afeta cerca de 1 bilhão de pessoas no mundo, constituindo um fator de risco independente para a doença cardiovascular (DCV) $)^{(3)}$. A hipertensão acomete cerca de $70 \%$ dos pacientes com diabetes mellitus tipo 2 (DM2) $)^{(5)}$. A morte de $80 \%$ dos diabéticos dá-se em decorrência de eventos trombóticos, sendo que $75 \%$ são eventos cardiovasculares ${ }^{(2)}$. A HAS pode ter um peso adicional sobre o DM, possivelmente agravando o quadro dos pacientes portadores das duas morbidades. Dessa forma, a investigação da contribuição da HAS nas alterações do perfil lipídico em indivíduos diabéticos pode abrir perspectivas para melhores controle e tratamento da doença. A morbidade e a mortalidade conseqüentes ao DM permanecem altas apesar do controle dos fatores de risco convencionais, sugerindo que a fisiopatologia dessa doença envolve outros fatores de grande importância clínica ${ }^{(14,35)}$. Portanto, a patogênese multifatorial do diabetes pode ser agravada pela associação entre hipertensão e fatores metabólicos, hemeostáticos e genéticos. Em virtude da alta prevalência de DM2 na população mundial e da elevada taxa de mortalidade decorrente de eventos aterotrombóticos, principalmente associados a DCV, é de extrema importância o conhecimento das alterações no perfil lipídico em pacientes portadores dessa doença.

As dislipidemias, o DM e a HAS são considerados importantes fatores no desenvolvimento da doença arterial coronariana (DAC), a principal causa de morte de indivíduos adultos no mundo ${ }^{(29,33)}$. Diversos estudos epidemiológicos têm demonstrado correlação positiva entre concentrações plasmáticas elevadas de LDL-C e/ou baixas concentrações de HDL-C e aumento de risco para DCV. Considerando a alta prevalência de indivíduos diabéticos hipertensos, o conhecimento das alterações bioquímicas neste grupo é importante para o melhor conhecimento de modificações que ocorrem na maior parcela dos indivíduos diabéticos. $\mathrm{O}$ impacto das doenças isoladas e de suas associações poderá ser mais bem compreendido se verificado o perfil lipídico nos diferentes grupos de indivíduos.

Nos indivíduos portadores de DM2 há forte associação entre microalbuminúria e risco de $D C V$, que é independente dos fatores de risco convencionais como hipertensão e tabagismo, sendo paradoxalmente um preditor de macroangiopatia $^{(6,8)}$.
A prevalência de microalbuminúria na população geral norte-americana atinge $28 \%$ em indivíduos com história de diabetes, $16 \%$ em hipertensos e $5,1 \%$ naqueles sem diabetes, hipertensão ou elevação da creatinina sérica ${ }^{(8)}$. Hirano et al. (2000) ${ }^{(11)}$, correlacionando fator von Willebrand solúvel (FvW) e trombomodulina solúvel (TM) - marcadores de lesão vascular - com albuminúria, observaram, em indivíduos diabéticos, que a dosagem da albuminúria não é somente um indicador de nefropatia, mas sim de lesão vascular sistêmica. A presença de microalbuminúria é então um preditor de agravamento da nefropatia diabética e um fator de risco independente para a $D C^{(6,31)}$. A prevenção da microalbuminúria no DM tipos 1 e 2 pode ser alcançada através de bom controle glicêmico e da pressão arterial com o uso de medicamentos anti-hipertensivos ${ }^{(31)}$.

Considerando o envolvimento das dislipidemias na gênese da aterosclerose, o presente estudo teve como objetivo correlacionar as concentrações plasmáticas dos lípides, das lipoproteínas, das apolipoproteínas e da microalbuminúria em pacientes adultos com e sem DM e HAS.

\section{Material e métodos}

Este estudo de coorte transversal avaliou 62 indivíduos, sendo 41 do sexo masculino, com faixa etária entre 40 e 65 anos, selecionados na Santa Casa de Misericórdia de Belo Horizonte (SCBH) através de critérios clínicos e laboratoriais que foram divididos em cinco grupos: 1. controle (indivíduos hígidos, $n=16$ ); 2 . HAS (hipertensos, $n=12$ ); 3. DM (DM2, normotensos e normoalbuminúricos, $n=7$ ); 4 . DM + HASnAlb (DM2, hipertensos e normoalbuminúricos, $n=18$ ); e 5. DM + HASmAlb (DM2, hipertensos e microalbuminúri$\cos , n=9$ ). Foram considerados diabéticos indivíduos com diagnóstico clínico prévio de DM2, com glicemia de jejum igual ou superior a $126 \mathrm{mg} / \mathrm{dl}^{(1)}$. Foram classificados como hipertensos indivíduos com pressão sangüínea igual ou superior a $140 \mathrm{mmHg}$ para a pressão arterial sistólica (PAS) ou $90 \mathrm{mmHg}$ para a pressão arterial diastólica (PAD), ou que estivessem fazendo uso de medicação anti-hipertensiva ${ }^{(3)}$. Indivíduos que apresentaram excreção urinária de albumina com valores superiores a $15 \mathrm{mg} / \mathrm{l}$ foram considerados microalbuminúricos. Os dados demográficos dos participantes estão representados na Tabela 1.

As amostras de sangue venoso foram obtidas com o paciente em jejum de 12 horas, utilizando-se tubos de vácuo sem anticoagulante. As amostras foram centrifugadas a 2.500rpm por 10 minutos para obtenção do soro. A determinação do CT e de TGL foi realizada pelo méto- 
Tabela 1 Caracterização dos grupos estudados

\begin{tabular}{lccccc}
\hline & Controle & HAS & DM & DM + HASnAlb & DM + HASmAlb \\
$n(\mathrm{M} / \mathrm{F})$ & $16(9 / 7)$ & $12(8 / 4)$ & $7(3 / 4)$ & $18(16 / 2)$ & $9(5 / 4)$ \\
Idade (anos) & $52,3 \pm 5,4$ & $54,8 \pm 5,2$ & $52,1 \pm 8,3$ & $57,2 \pm 6$ & $55,1 \pm 6,2$ \\
IMC $\left(\mathrm{kg} / \mathrm{m}^{2}\right)$ & $25,1 \pm 3,9$ & $28,4 \pm 3,6$ & $28,1 \pm 3,5$ & $28,6 \pm 4,1$ & $29,7 \pm 3,9$ \\
\hline
\end{tabular}

Caracterização quanto a n (tamanho), M (sexo masculino) e de F (sexo feminino), idade e índice de massa corporal (IMC), sendo os valores dos dois últimos parâmetros expressos como média e desvio-padrão.

do enzimático colorimétrico utilizando-se os reagentes Randox ${ }^{\circledR}$ Cholesterol CHOD-PAP e Randox ${ }^{\circledR}$ Triglycerides GPO-PAP, respectivamente. A estimativa dos valores de LDL-C foi feita utilizando-se a equação de Friedewald. O HDL-C foi quantificado pelo método enzimático homogêneo utilizando-se os reagentes Randox ${ }^{\circledR} \mathrm{HDL}$ Cholesterol Direct. As determinações de ApoB e de ApoA-I foram realizadas por turbidimetria, utilizando-se os reagentes Biotécnica ${ }^{\circledR}$ Apolipoproteína B Turbidimetria e Biotécnica Apolipoproteína A-I Turbidimetria, respectivamente. A determinação da lipoproteína(a) foi feita utilizando-se os reagentes In Vitro ${ }^{\circledR}$ Lipoproteína(a) Turbidimétrico. Todas as dosagens foram realizadas no aparelho Cobas Mira Plus ${ }^{\circledR}$, utilizando-se soros-controle para verificar o desempenho dos ensaios. Na análise estatística, o teste de análise de variância (ANOVA) foi utilizado para os parâmetros $C T$, ApoA-I, ApoB, HDL-C e LDL-C, que apresentaram distribuição normal e homocedasticidade. Especialmente para os parâmetros TCL e $L p(a)$, a análise de variância foi realizada pelo método Student-Newman-Keuls, após transformação logarítmica dos dados. Foi realizado o teste de correlação
Pearson, considerando-se todos os indivíduos estudados. Valor de $p$ menor que 0,05 foi considerado estatisticamente significante. O programa Sigma Stat versão 1.0 foi utilizado para realizar as análises.

\section{Resultados}

Os resultados obtidos das análises bioquímicas realizadas estão demonstrados na Tabela 2. Foram observadas diferenças estatísticas para o LDL-C entre a média do grupo $\mathrm{DM}+\mathrm{HASmAlb}$ em relação às médias dos demais grupos ( $p$ $<0,0001)$; para TGL entre os grupos HAS, DM + HASnAlb e DM + HASmAlb em relação ao grupo controle $(p<0,009$, $p<0,0004, p<0,0001$, respectivamente). Para CT, HDL-C e $L p(a)$ não houve diferenças significativas entre as médias dos grupos. $O$ valor das médias para ApoB dos grupos DM + HASnAlb e DM + HASmAlb apresentaram diferenças significativas com relação ao grupo controle $(p<0,001, p$ $<0,004$, respectivamente), entretanto, para a ApoA-I não houve diferença estatística entre os grupos. Houve corre-

\section{Tabela 2 Dados laboratoriais dos grupos em estudo}

\begin{tabular}{lcccccc} 
mg/dl & Controle & HAS & DM & DM + HASnAlb & DM + HASmAlb & Valor de $p$ \\
& $n=16$ & $n=12$ & $n=7$ & $n=18$ & $n=9$ & \\
CT & $180,4 \pm 27,9$ & $198,4 \pm 44,5$ & $201,7 \pm 44,2$ & $190,6 \pm 44,5$ & $218,4 \pm 38,2$ & ns \\
HDL-C & $47,5 \pm 10,15$ & $40 \pm 13,2$ & $41 \pm 7,02$ & $39,6 \pm 11,95$ & $40,8 \pm 16,32$ & ns \\
LDL-C & $116,3 \pm 29,8$ & $129,9 \pm 39,7$ & $137,4 \pm 46$ & $117,5 \pm 37$ & $210,6 \pm 63,5^{\mathrm{a}, \mathrm{b}, \mathrm{c}, \mathrm{d}}$ & $p^{\mathrm{a}, \mathrm{b}, \mathrm{c}, \mathrm{d}}$ \\
& & & & & $<0,0001$ \\
TGL & $82,9 \pm 45,2$ & $142,5 \pm 69,2^{\mathrm{a} 1}$ & $116,7 \pm 53,1$ & $168,0 \pm 75^{\mathrm{a} 2}$ & $164,4 \pm 101,9^{\mathrm{a} 3}$ & $\mathrm{p}^{\mathrm{a} 1}<0,01$ \\
& & & & & $p^{\mathrm{a} 2, \mathrm{a} 3}<0,001$ \\
Lp(a) & $25,8 \pm 26$ & $18,4 \pm 17,4$ & $15,3 \pm 15,8$ & $35,3 \pm 29,6$ & $19,8 \pm 15$ & $\mathrm{~ns}$ \\
Apo A-I & $133,2 \pm 7,53$ & $132,8 \pm 9,61$ & $135,7 \pm 11,58$ & $136 \pm 10,19$ & $134,7 \pm 8,3$ & $\mathrm{~ns}$ \\
Apo B & $64,5 \pm 10,8$ & $72,5 \pm 13,6$ & $75,8 \pm 18,3$ & $78,9 \pm 12,1^{\mathrm{a} 1}$ & $83,3 \pm 13,5^{\mathrm{a} 2}$ & $p^{\mathrm{a} 1, \mathrm{a} 2}<0,01$ \\
\hline
\end{tabular}

Os dados estão representados pelas médias e desvios-padrão dos valores plasmáticos de CT, HDL-C, LDL-C, TCL, LP(a), Apo A-I e Apo B para os grupos controle, HAS (com hipertensão), DM (com DM2), DM + HASnAlb (com DM2 normoalbuminúrico e hipertensão) e DM + HASmAlb (com DM2 microalbuminúrico e hipertensão). Valores expressos em mg/dl. As diferenç̧as significativas entre os grupos estão representadas pelas letras: a. vs. controle; b. vs. HAS; c. vs. DM e d. vs. DM + HASnAlb. 
lação positiva entre ApoB e LDL-C $(r=0,68$ e $p<0,0001)$ e entre ApoA-I e HDL-C $(r=0,37$ e $p=0,003)$.

\section{Discussão}

A hiperlipidemia foi definida no passado como níveis plasmáticos de TGL e colesterol excedendo os normais, que em geral têm sido arbitrariamente definidos como o 95 - percentil dos níveis da população em geral. Entretanto agora tornou-se evidente que os níveis plasmáticos ideais ou ótimos de colesterol são muito inferiores aos normais da população geral, e que o risco de DAC aumenta à medida que os níveis de CT se elevam acima dos valores ideais ${ }^{(34)}$. A manutenção dos níveis plasmáticos ideais de lípides constitui um dos objetivos do tratamento dos indivíduos diabéticos justamente para evitar a progressão das complicações macrovasculares secundárias ao diabetes ${ }^{(33)}$. Com relação ao CT não foram observadas diferenças significativas entre as médias dos grupos avaliados, entretanto o grupo DM + HASmAlb apresentou a média mais alta em relação aos outros grupos (Tabela 2), sugerindo que a presença da microalbuminúria poderia estar correlacionada com a dislipidemia.

As médias para a fração LDL-C dos grupos DM e DM + HASnAlb e DM + HASmAlb encontram-se acima dos valores ideais para diabéticos $(<100 \mathrm{mg} / \mathrm{dl})^{(28)}$. No grupo DM + HASmAlb, os níveis de LDL-C apresentam-se elevados, cerca de duas vezes maiores que o valor recomendável para os diabéticos ${ }^{(28)}$, caracterizando, neste grupo, aumento de risco cardiovascular. Além disso, uma outra preocupação em relação à fração LDL-C no diabético é que as diferenças parecem ser mais qualitativas do que quantitativas. Verifica-se na literatura a indicação de uma alta prevalência de partículas pequenas e densas em indivíduos diabéticos, mesmo quando normolipêmicos ${ }^{(19)}$. No presente estudo foram observadas diferenças significativas para a fração LDLC entre o grupo DM + HASmAlb e os demais, sugerindo que a presença da microalbuminúria favorece a dislipidemia.

Para o HDL-C não foi observada diferença significativa entre as médias dos grupos, porém os valores médios dos grupos DM, DM + HASnAlb e DM + HASmAlb estão abaixo dos valores desejáveis (> 40mg/dl para não-diabéticos e $>45 \mathrm{mg} / \mathrm{dl}$ para diabéticos) $)^{(28)}$.

Com relação aos TGL as diferenças entre as médias foram significativamente mais elevadas nos grupos HAS, DM + HASnAlb e DM + HASmAlb em relação à média do grupo controle. Deve-se ressaltar que esses grupos têm em comum o aumento da pressão arterial, importante fator de risco para DCV. Apenas as médias dos grupos DM + HASnAlb e DM + HASmAlb encontram-se ligeiramente acima dos valores desejáveis $(<150 \mathrm{mg} / \mathrm{dl})^{(28)}$, demonstrando nesses grupos a persistência de pequena elevação dos TGL apesar do controle da dieta e/ou da adoção de medidas farmacológicas. Outra possível explicação para esse achado é a associação das duas morbidades, o que pode estar agravando a dislipidemia por sinergismo.

Está bem documentada na literatura a existência de uma correlação direta entre os níveis plasmáticos de $\mathrm{CT}$, assim como entre os níveis de LDL-C e de DCV. Ademais, ainda existe uma correlação inversa entre níveis plasmáticos de HDL-C e o risco dessas doenças. Entretanto, ao contrário da numerosa literatura disponível para o risco de DCV, a correlação entre anormalidades apolipoprotéicas ainda não está bem consolidada em pacientes diabéticos e hipertensos.

Nesse estudo, na avaliação do perfil apolipoprotéico, não foram observadas diferenças estatisticamente significativas para a ApoA-l entre as médias dos cinco grupos. Para o parâmetro ApoB as médias dos grupos DM + HASnAlb e DM + HASmAlb apresentaram-se significativamente diferentes em relação ao grupo controle. Entretanto os valores das médias desses parâmetros situam-se na faixa de valores de referência para apolipoproteínas, demonstrando que a maioria dos indivíduos selecionados não apresentou alterações significativas no perfil apolipoprotéico. Torna-se oportuno ressaltar que o grupo DM + HASmAlb apresentou níveis plasmáticos significativamente mais elevados de LDL-C em relação aos demais grupos, seguido pelo grupo DM + HASnAlb.

Apesar de os valores médios de ApoB se encontrarem dentro da faixa de referência nos grupos DM + HASnAlb e DM + HASmAlb, estes são significativamente mais elevados em relação ao grupo controle, demonstrando que a associação das duas patologias pode ter influenciado os níveis plasmáticos do citado parâmetro.

As correlações positivas observadas entre HDL-C e ApoA-I $(r=0,37 ; p=0,003)$ e entre LDL-C e ApoB $(r=0,68 ; p<0,0001)$ já eram esperadas, uma vez que a principal apolipoproteína constituinte da lipoproteína de alta densidade (HDL) é a ApoA-I, e da lipoproteína de baixa densidade (LDL), a ApoB. Entretanto, estabelecer a magnitude da correlação torna-se importante para a validação dos testes que não são empregados rotineiramente pelos laboratórios clínicos, como é o caso das dosagens de ApoA-I e de Apo B para avaliar o perfil lipídico de pacientes diabéticos. Os níveis plasmáticos de apolipoproteínas têm sido descritos como melhores preditores de DCV do que as lipoproteínas ${ }^{(12,32)}$. As concentrações de apolipoproteínas 
são geneticamente determinadas e sofrem pouca influência de variáveis biológicas, enquanto que os lípides apresentam flutuação dos seus níveis plasmáticos em torno de seus pontos de equilíbrio ou homeostáticos em resposta aos vários estímulos do controle metabólico ${ }^{(18)}$. As partículas aterogênicas de LDL e os remanescentes de quilomícrons e lipoproteína de muito baixa densidade (VLDL) apresentam uma molécula de ApoB como parte da estrutura protéica. Por isso, a concentração plasmática de $A p o B$ tem sido considerada um melhor representante das partículas aterogênicas ou do colesterol não-HDL, e alguns estudos têm demonstrado que níveis plasmáticos elevados de $A p o B$ podem ser um valioso preditor de $\operatorname{DAC}^{(26)}$.

Para o parâmetro $L p(a)$ não foram observadas diferenças significativas entre as médias dos cinco grupos. Entretanto, o grupo DM + HASnAlb apresentou média de Lp(a) acima dos valores de referência (até $30 \mathrm{mg} / \mathrm{dl}$ ). A Lp(a) apresenta um caráter duplamente aterogênico devido ao fato de apresentar composição lipídica similar à LDL e à presença da apolipoproteína(a) em sua estrutura, proteína que apresenta alto grau de homologia com o plasminogênio. Isso resulta em comprometimento da eficiência do sistema fibrinolítico, já que a $L p(a)$ pode competir com o plasminogênio, o qual constitui o precursor inativo da plasmina, enzima responsável pela quebra da fibrina produzida no processo da coagulação ${ }^{(15)}$. O possível papel dos níveis plasmáticos de $\mathrm{Lp}(\mathrm{a})$ no desenvolvimento de DAC em pacientes diabéticos é controverso. Alguns estudos demonstram a associação direta entre níveis plasmáticos de $L p(a)$ e o desenvolvimento de $\operatorname{DAC}(4,10,13,21,22,27)$, enquanto outros demonstram o contrário(9, 23-25). Gazzaruso et al. (2001) $)^{(7)}$ demonstraram que os níveis plasmáticos de $L p(a)$ foram independentemente associados com DAC em pacientes com DM, entretanto os níveis dessa lipoproteína comportaram-se como fator de risco cardiovascular menos importante em pacientes diabéticos do que em indivíduos não-diabéticos. Em um estudo recentemente realizado em nosso laboratório(16), os níveis de $\mathrm{Lp}(\mathrm{a})$ se correlacionaram com a forma clínica da DAC, considerando que $72 \%$ dos pacientes não-diabéticos com forma grave de ateromatose apresentaram níveis elevados de $L p(a)$ contra apenas $22 \%$ de pacientes com a forma leve/moderada confirmadas angiograficamente. Os dados da literatura, aliados aos nossos, permitem aventar a hipótese de que a possível glicação da $L p(a)$ em pacientes diabéticos contribuiria para menor risco de DCV. Essa glicação resultaria numa molécula com menor homologia com o plasminogênio. Dessa forma, o desempenho do sistema fibrinolítico seria menos afetado neste grupo de pacientes, ou seja, a fibrina formada seria eficientemente degradada por este sistema, não favorecendo o desenvolvimento de DCV em pacientes diabéticos.

O presente estudo demonstra ausência de associação entre níveis plasmáticos de $L p(a)$ e a presença isolada ou combinada das duas patologias estudadas (HAS e DM), uma vez que não foram observadas diferenças significativas entre os grupos estudados para o referido parâmetro. No entanto, esse estudo demonstrou a presença de níveis médios de Lp(a) elevados no grupo DM + HASnAlb, sugerindo que a associação das duas patologias poderia ter contribuído para a elevação de $L p(a)$ nesse grupo. Entretanto esse fato não ocorreu no grupo DM + HASmAlb. Os níveis plasmáticos de Lp(a) e a sua massa molecular são muito variáveis entre as pessoas, sendo determinados geneticamente ${ }^{(17)}$. Contudo certas anormalidades metabólicas podem influenciar as concentrações circulantes de $L p(a)$. Esses valores podem ser aumentados como parte de uma resposta de fase aguda, no DM, insuficiência renal crônica, síndrome nefrótica, câncer, menopausa e hipotireoidismo ${ }^{(20)}$. No entanto, os nossos dados não concordam com a idéia de que o DM em si poderia contribuir para o achado de níveis elevados de $L p(a)$. A controvérsia existente na literatura em relação à associação dos níveis plasmáticos de $L p(a)$, e o desenvolvimento de DAC em pacientes diabéticos poderia se justificar pela grande heterogeneidade de co-morbidades existente em pacientes diabéticos.

As dislipidemias, o DM e a hipertensão arterial são reconhecidamente fatores de risco para a DAC. Portanto torna-se importante um maior conhecimento das associações entre esses fatores para a orientação e a adoção de medidas preventivas e terapêuticas na monitorização do DM. A exemplo da reconhecida importância da investigação da microalbuminúria como preditor da macroangiopatia e do agravamento da nefropatia diabética, outros fatores também devem ser investigados, ampliando, assim, as possibilidades de uma melhor monitorização do paciente diabético.

\section{Agradecimentos}

Ao Prof. Dr. Ivan Barbosa Machado Sampaio, pelo auxílio na análise estatística. À Fundação de Amparo à Pesquisa de Minas Gerais (FAPEMIG), à Fundação Coordenação de Aperfeiçoamento de Pessoal de Nível Superior(CAPES), ao Conselho Nacional de Desenvolvimento Científico e Tecnológico (CNPq), ao LABORATEL Laboratório de Análises Clínicas e à BIOTÉCNICA Biotecnologia Avançada, pelo apoio recebido. 


\section{Referências}

I. ADA (AMERICAN DIABETES ASSOCIATION). Follow-up report on the diagnosis of diabetes mellitus. Diabetes Care, v. 26, 2003.

2. CARR, M.E. Diabetes mellitus: a hypercoagulate state.J Diabetes Com, v. I5, n. I, p. 44-54, 200 I.

3. CHOBANIAN,A.V. et al. National High Blood Pressure Education Program Coordinating Committee. The seventh report of the Joint National Committee on prevention, detection, evaluation, and treatment of high blood pressure: The JNC 7 report. JAMA, v. 289, n. 19, p. 2560-72, 2003.

4. COMLEKCl, A. et al. Correlation between serum lipoprotein(a) and angiographic coronary artery disease in non-insulindependent diabetes mellitus. J Int Med, v. 242, p. 449-54, 1997.

5. DODSON, P.M. Hypertension and diabetes. Curr Med Res Opin, v. 18, n. I, p. 48-57, 2002

6. FIORETTO, P.et al. Heterogeneous nature of microalbuminuria in NIDDM: studies of endothelial function and renal structure. Diabetologia, v. 4I, p. 233-36, 1998.

7. GAZZARUSO, C. et al. Association of lipoprotein(a) levels and apolipoprotein(a) phenotypes with coronary artery disease in type 2 diabetic patients and in non-diabetic subjects Diabetic Medicine, v. 18, p. 589-94, 2001.

8. GIORGI, D.M.A. Microalbuminúria na hipertensão arterial primária: significado e valor prognóstico. Rev Soc Cardiol, v. I3, n. I, p. 85-91, 2003.

9. HAFFNER, S.M. et al. Lack of association between lipoprotein(a) concentrations and coronary heart disease mortality in diabetes: the Wisconsin epidemiologic study of diabetic retinopathy. Metabolism, v. 4I, p. 194-7, 1992.

10. HEESEN, B.J. et al. Lipoprotein(a) levels in relation to diabetic complications in patients with non-insulin-dependent diabetes. Eur J Clin Invest, v. 23, p. 580-84, 1993.

I I. HIRANO,T. et al.Vascular endothelial markers, von Willebrand factor and thrombomodulin index, are specifically elevated in type 2 diabetic patients with nephropathy: comparison of primary renal disease. Clin Chim Acta, v. 299, n. 1-2, p. 65-75, 2000.

12. HSIA, S.L. et al. Serum levels of high-density lipoprotein phospholipids correlate inversely with severity of angiographically defined coronary artery disease. Atherosclerosis, v. 152, p. 469-73, 2000.

13. JAMES, R.W. et al. Lipoprotein(a) and vascular disease in diabetic patients. Diabetologia, v. 38, p. 7। I-4, 1995.

14. JUHAN-VAGUE, I. et al. Thrombogenic and fibrinolytic factor and cardiovascular risk in non-insulin-dependent diabetes mellitus. Ann Med, v. 28, n. 4, p. 37I-80, 1996.

15. KOSCHINSKY, M.L. Lipoprotein(a) and the link between atherosclerosis and thrombosis. Can J Cardiol, v. 20, n. 8, p. 37B-43B, 2004. Suplemento B.

16. LIMA, L.M. Perfil lipídico e parâmetros hemostáticos em indivíduos submetidos à angiografıa coronariana. 2005. I 03f. Dissertação
(Mestrado em Ciências Farmacêuticas). Faculdade de Farmácia, Universidade Federal de Minas Gerais, Belo Horizonte.

17. MARCOVINA, S.M. et al. Report of The National Heart, Lung, and Blood Institute Workshop on Lipoprotein(a) and Cardiovascular Disease: recent advances and future directions. Clin Chem, v. 49, p. 1785-96, 2003.

I8. MARTINEZ, T.L.R. Dislipidemias: da teoria à prática. São Paulo: Atheneu, 2004. 416p.

19. MARTINEZ,T.L.R. Manual de condutas clínicas em dislipidemias. Rio de Janeiro: Medline, 2003. 392p.

20. MILIONIS, H.J. et al. Lipoprotein(a) and stroke. J Clin Pathol, v. 53, p. 487-96, 2000.

2I. MOHAN, V. et al. Lipoprotein(a) is an independent risk factor for coronary artery disease in NIDDM patients in South India. Diabetes Care, v. 21, p. 1819-23, 1998.

22. MURAKAMI, K. et al. Lipoprotein(a) as a coronary risk factor in Japanese patients with type II (non-insulin-dependent) diabetes mellitus. Relation with apolipoprotein(a) phenotypes. Diabetologia, v. 4I, p. 1397-9, 1998.

23. NISKANEN, L. et al. Apoprotein(a) levels in relation to coronary heart disease and risk factors in type II (noninsulin-dependent) diabetes. Cardiovasc Risk Factors, v. 13 , p. 205-10, 1993.

24. O'BRIEN,T. et al. Lipids and Lp(a) lipoprotein levels and coronary artery disease in subjects with non-insulin-dependent diabetes mellitus. Mayo Clin Proc, v. 69, p. 430-5, 1994.

25. RELIMPIO, F. et al. Lack of association of lipoprotein(a) with coronary heart disease in Spaniard type 2 diabetic patients. Diabetes Res Clin Pract, v. 35, p. I35-4I, 1997.

26. ROETERS VAN LENNEP, J.E. et al. Apolipoprotein concentrations during treatment and recurrent coronary artery disease events. Arterioscler Thromb Vasc Biol, v. 20, p. 2408-13, 2000.

27. RUIZ, J. et al. Association of elevated lipoprotein(a) levels and coronary heart disease in NIDDM patients. Relationship with apolipoprotein(a) phenotypes. Diabetologia, v. 37, p. 585-91, 1994.

28. SBC (SOCIEDADE BRASILEIRA DE CARDIOLOGIA). III Diretrizes Brasileiras Sobre Dislipidemias e Diretrizes de Prevenção da Aterosclerose. Arq Bras Cardiol, v. 77, p. I-48, 200 I. Suplemento III.

29. SBD (SOCIEDADE BRASILEIRA DE DIABETES). Consenso Brasileiro sobre Diabetes. Diagnóstico e classificação do diabetes mellitus e tratamento do diabetes mellitus tipo 2,2000. Disponível em: <http://www.diabetes.org.br/consenso/ index.html>. Acesso em: 27 set. 2002.

30. STEHOUWER, C.D.A. et al. Increased urinary albumin excretion, endothelial dysfunction, and chronic Low-Grade Inflammation In Type 2 Diabetes: prospective, interrelated, and independently associated with risk of death. Diabetes, v. 5I, n. 4, p. I |57-65, 2002. 
31.TOBE, S.W. et al. Can Med J, v. 167, n. 5, p. 499-503, 2002.

32. WESTERVELD, H.T. et al. Apolipoprotein B and coronary artery disease in women. A cross-sectional study in women undergoing their first coronary angiography. Arterioscler Thromb Vasc Biol, v. 18, p. II01-7, 1998.

33.WHO (WORLD HEALTH ORGANIZATION). Diabetes mellitus. Fact sheets, n. 1 38,2002. Disponível em: <http://www.who.int/ inf-fs/en/fact I 38.html>. Abril 2002. Acesso em: 27 set. 2004
34. WITZTUM, J.L. Fármacos usados no tratamento das hiperlipoproteinemias. In: HARDMAN, J.G.; LIMBIRD, L.E. (Ed.). Goodman \& Gilman: as bases farmacológicas da terapêutica. 9. ed. Rio de Janeiro: McGraw Hill, 1996. cap. 36, p. 643-60

35. YAMADA, T. et al. Importance of hypercoagulability over hyperglycemia for vascular complication in type 2 diabetes. Diabetes Res Clin Prac, v. 49, n. I, p. 23-31, 2000. 